\title{
Prospective study on prevalence, intensity, type, and therapy of acute pain in a second-level urban emergency department
}

This article was published in the following Dove Press journal:

Journal of Pain Research

\author{
Paolo Mura' \\ Elisabetta Serra' \\ Franco Marinangeli ${ }^{2}$ \\ Sebastiano Patti ${ }^{3}$ \\ Mario Musu' \\ Ilenia Piras ${ }^{3}$ \\ Maria Valeria Massidda' \\ Giorgio $\mathrm{Pia}^{3}$ \\ Maurizio Evangelista ${ }^{4}$ \\ Gabriele Finco' \\ 'Department of Medical Sciences "M. \\ Aresu", University of Cagliari, Cagliari, \\ Italy; ${ }^{2}$ Department of Anesthesiology, \\ Intensive Care and Pain Medicine, \\ University of L'Aquila, L'Aquila, Italy; \\ ${ }^{3}$ Department of Emergency Medicine, \\ Santissima Trinità Hospital, Cagliari, \\ Italy; ${ }^{4}$ Department of Anesthesiology \\ and Pain Medicine, Cattolica \\ University, Rome, Italy
}

\begin{abstract}
Aim: Pain represents the most frequent cause for patient admission to emergency departments (EDs). Oligoanalgesia is a common problem in this field. The aims of this study were to assess prevalence and intensity of pain in patients who visited a second-level urban ED and to evaluate the efficacy of pharmacological treatment administered subsequent to variations in pain intensity. Methods: A 4-week prospective observational study was carried out on 2,838 patients who visited a second-level urban ED. Pain intensity was evaluated using the Numeric Rating Scale at the moment of triage. The efficacy of prescribed analgesic therapy was evaluated at 30 and 60 minutes, and at discharge. Data concerning pain intensity were classified as absent, slight, mild, or severe. Pain was evaluated in relation to the prescribed therapy.
\end{abstract}

Results: Pain prevalence was $70.7 \%$. Traumatic events were the primary cause in most cases (40.44\%), followed by pain linked to urologic problems (13.52\%), abdominal pain (13.39\%), and nontraumatic musculoskeletal pain (7.10\%). Only $32.46 \%$ of patients were given pharmacological therapy. Of these, $76 \%$ reported severe pain, $19 \%$ moderate, and 5\% slight, and $66 \%$ received nonsteroidal anti-inflammatory drugs or paracetamol, $4 \%$ opioids, and $30 \%$ other therapies. A difference of at least 2 points on the Numerical Rating Scale was observed in $84 \%$ of patients on reevaluation following initial analgesic therapy.

Conclusion: Pain represents one of the primary reasons for visits to EDs. Although a notable reduction in pain intensity has been highlighted in patients who received painkillers, results show that inadequate treatment of pain in ED continues to be a problem.

Keywords: pain, emergency department, oligoanalgesia, pain treatment, pain assessment, NSAIDs, opioids, methoxyflurane, traumatic pain, abdominal pain

\section{Introduction}

Pain is one of the most common symptoms mentioned by patients at emergency departments (EDs). ${ }^{1,2}$ Painful conditions cause over $70 \%$ of all ED visits in the US and Canada. ${ }^{3}$ Chang et $\mathrm{al}^{4}$ reported that from 2000 to 2010 in the United States, $\sim 45.4 \%$ of ED visits were associated with a primary symptom or diagnosis of pain.

Previous studies reported that many patients suffering from acute pain receive inadequate or no treatment at ED. ${ }^{5,6}$ Despite this, studies on the prevalence of pain in EDs are still scarce. ${ }^{1,2,7}$ In addition, pain often represents an underestimated and undertreated problem in Italy. ${ }^{8}$

In 1989, Wilson and Pendleton coined the term "oligoanalgesia" to describe the lack of adequate treatment of pain in terms of dosages and rapidity of administration of analgesics for ED patients. ${ }^{9}$ To date, no recognized universal instruments are
Correspondence: Gabriele Finco Department of Medical Sciences "M. Aresu", University of Cagliari, SS554 Bivio Sestu - Monserrato, Cagliari, Italy Tel +39070510965I3

Email gabriele.finco@gmail.com 
available to define expectations and outline objectives of pain relief. Available instruments and pain evaluation tables all beg the question "What does a score of $\mathrm{x}$ on the scale of pain mean exactly?"

Results obtained by Farrar ${ }^{10}$ in his study to determine the limits to evaluate important variations in pain show that a 2-point difference on the Numerical Rating Scale (NRS) scale is significant.

Pain therapy should be parallel to reaching diagnosis. Pain is considered the fifth vital sign, and thus, it should be evaluated at a patient's visit to the $\mathrm{ED}^{11}$ and reevaluated once therapy has been administered. Most used drugs in everyday ED clinical practice are nonsteroidal anti-inflammatory drugs (NSAIDs) - especially ibuprofen, ketoprofen, and ketorolacand paracetamol. They can be administered either enterally or parenterally. Tramadol or opioids such as morphine, hydromorphone, or fentanyl can also be used. Association of analgesics with adjuvant nonanalgesic drugs can improve their effects or mitigate adverse side effects.

Pain evaluation is important, and it is used as a quality indicator. $^{12,13}$

Literature emphasizes the need to shift research attention from the prompt diagnosis of the underlying condition of pain toward that of immediate pain relief, attempting to identify therapies that lead to a ready and efficient analgesic which is as side effect-free as possible. . $^{5,14}$

It is critical to treat acute pain not only to decrease suffering but also to maximize healing and minimize the chances of progression to a chronic pain condition. ${ }^{5}$

\section{Methods}

This prospective observational study was performed in accordance with the Good Clinical Practice (GPC) Guidelines, the EU CT Directive 2001/20.EC, GCP Commission Directive 2005/28/EC, and the Declaration of Helsinki (2008). The study was referred to the Cagliari Teaching Hospital Independent Ethical Committee that approved it as a low-intervention observational study. The Ethical Committee reviewed the study and established the exemption from inclusion in the National Observational Study Register. This study was performed in a second-level ED in Cagliari (Italy) to which about 35,000 patients are referred annually. Data on patients who accessed the ED were collected over a 4-week period. The primary study aim was to observe and describe the access of patients who complained of pain at the moment of triage, comparing the data obtained with those available in literature. The secondary aim was to evaluate the pharmacological therapies through the variations of pain from admission to the ED to discharge, during hospitalization, or at home.

The study enrolled consecutively patients aged 18 , registered at the triage of the ED during the study period. A written informed consent was obtained from all participants. Exclusion criteria were all conditions that rendered data collection impossible or only partially possible: nonconsent by the patient, alterations in states of consciousness (coma, drunkenness, psychomotor agitation, intoxication), cases of sexual abuse or domestic violence, prisoners, or conditions that led to the patient being referred directly for medical observation. The study collected demographic data (age and gender), the main reason for ED access, and element to assess patient pain by using the 11-point evaluation scale or NRS (Figure 1).

Each patient was asked if he or she could evaluate his or her level of pain on a scale from 0 to 10 , where 0 equaled an absence of pain and 10 the maximum level. Pain intensity evaluated at the moment of triage was indicated as " $t_{0}$ ". All analgesic therapies administered to patients and their clinical efficacy were evaluated by ED personnel. Pain levels were reevaluated 30 minutes after administration of therapy $\left(t_{1}\right)$ at 60 minutes, if a second dose was required $\left(\mathrm{t}_{2}\right)$, and in all patients treated at discharge $\left(t_{3}\right)$. Any side effects correlated to the analgesic drugs delivered were recorded.

Collected data were then stored in an Access database with security code protection.

Although translating continuous measures such as NRS into discrete categories is not straightforward, ${ }^{18}$ for the benefit of the analysis, data on pain intensity reported by patients at triage $\left(\mathrm{t}_{0}\right)$ with the 11-point NRS were classified into four categories: no pain (score $=0$ ), slight pain (score $1-3$ ), moderate pain (score 4-6), and severe pain (7-10), ${ }^{8}$ as shown in Table 1.

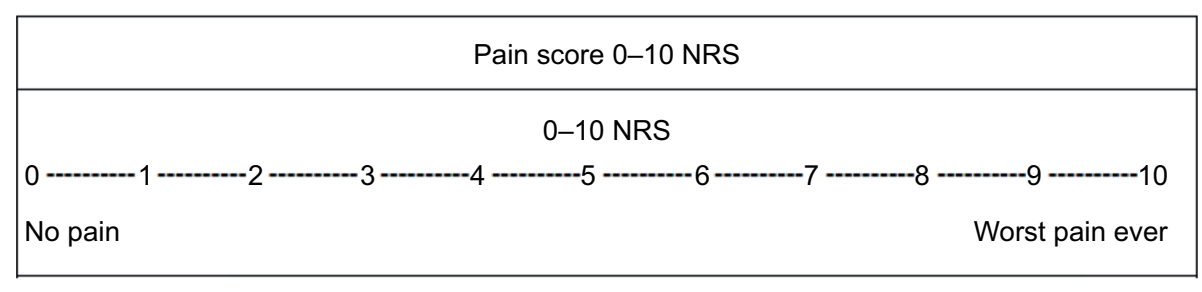

Figure I The Numerical Rating Scale (NRS). 


\section{Results}

During the study period, a total of 2,838 patients accessed the ED. At triage, 1,035 patients were enrolled. The other 1,803 were excluded because they did not satisfy protocol criteria.

Table I NRS score at triage in all interviewed patients $(\mathrm{N}=1,035)$

\begin{tabular}{llll}
\hline Category & $\begin{array}{l}\text { NRS } \\
\text { score }\end{array}$ & Patients, n & $\begin{array}{l}\text { Patients in category, } \\
\text { n (\%) }\end{array}$ \\
\hline No pain & 0 & 303 & $303(29.27 \%)$ \\
Slight pain & 1 & 17 & $60(5.8 \%)$ \\
& 2 & 15 & \\
Moderate pain & 4 & 48 & $223(21.55 \%)$ \\
& 5 & 62 & \\
Severe pain & 6 & 120 & $449(43.38 \%)$ \\
& 7 & 173 & \\
& 8 & 141 & \\
& 9 & 78 & \\
& 10 & 57 & \\
\hline
\end{tabular}

Abbreviation: NRS, Numerical Rating Scale.
Of the 1,035 enrolled subjects, $732(70.7 \%)$ reported a painful symptomatology, while the remaining $303(29.3 \%)$ reported no pain.

Of the 732 patients who reported pain, $48(6.56 \%)$ declined medical examination; the other 684 (93.44\%) accessed the medical center and $222(32.46 \%)$ were treated (Figure 2).

Analysis of the data on the 732 patients who reported pain at the moment of triage $\left(\mathrm{t}_{0}\right)$ revealed the presence of slight pain in $60(8.20 \%)$, moderate pain in $223(30.46 \%)$, and severe pain in $449(61.34 \%)$ patients (Table 1).

From the analysis of pain prevalence at $t_{0}(n=732)$, the highest number of accesses was due to traumatic events $(n=296 ; 40.44 \%)$, urological pathologies $(n=99 ; 13.52 \%)$, abdominal pain $(\mathrm{n}=98 ; 13.39 \%)$, and nontraumatic musculoskeletal pain ( $\mathrm{n}=52 ; 7.10 \%)$ (Table 2$)$.

Of the patients who were prescribed analgesic therapy, 6 $(2.70 \%)$ left before conclusion of the diagnostic therapeutic

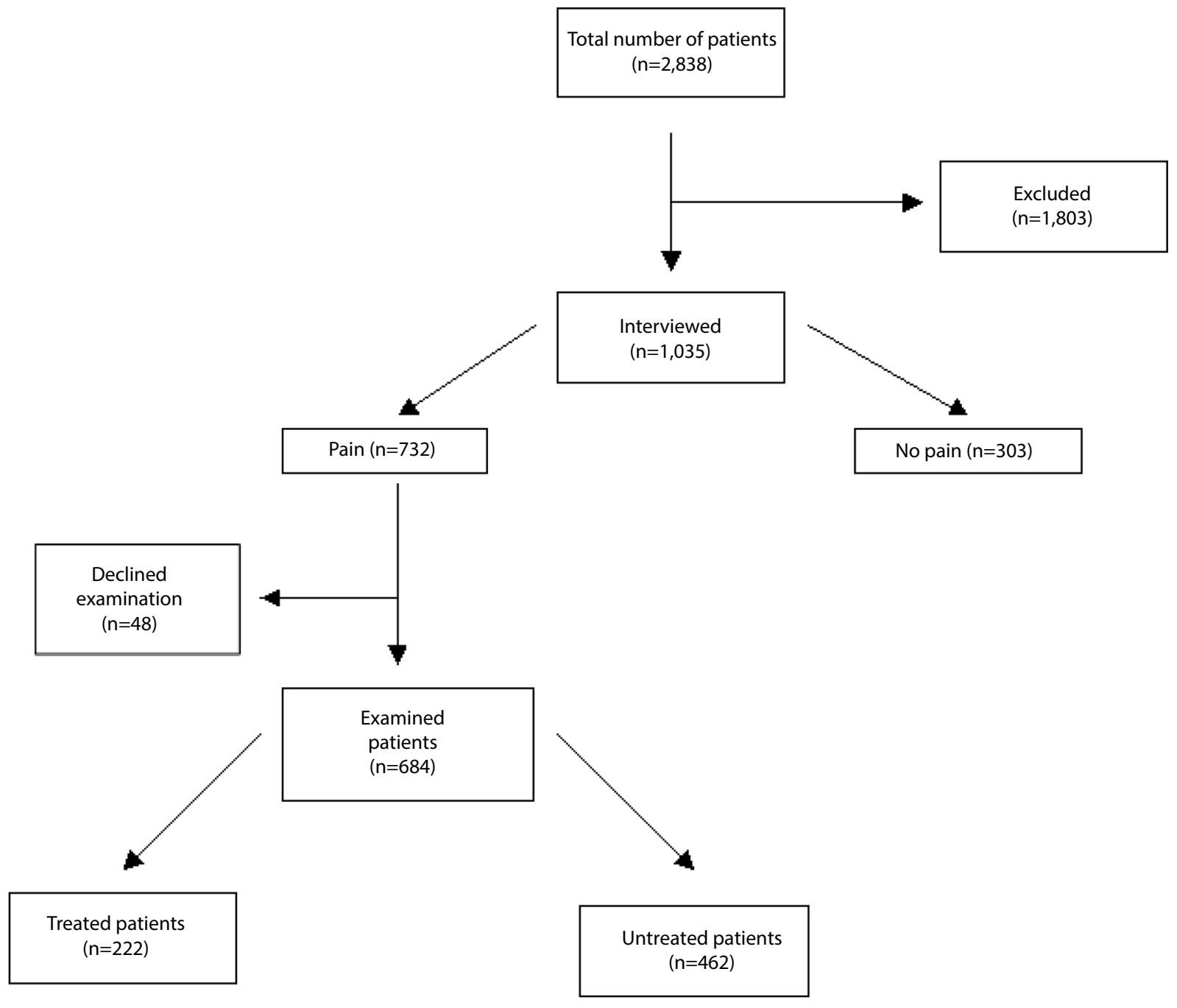

Figure 2 Number of patients enrolled in the study. 
evaluation, and so it was not possible to assess outcome on discharge.

The average age of treated patients was $45.69 \pm 18.35$ years (range $18-88$ years); $52 \%(n=115)$ were male and the remaining $48 \%(n=107)$ female; no significant difference in average NRS scores between males (mean NRS 7.30) and females (mean NRS 7.55) was observed.

Trauma victims and patients with urological (eg, renal colic, bladder infections, etc), abdominal (eg, appendicitis, cholecystitis, etc), and nontraumatic musculoskeletal pain (neck pain, low back pain, etc) reported higher scores on the NRS, with a mean of 6.08 (median =6), 7.24 (median $=7$ ), 7.44 (median $=8$ ), and 7.56 (median $=8)$, respectively.

Two hundred and twenty-two (32.46\%) of the 684 examined patients received analgesics (Table 3 ). The initial pharmacological treatment of painful symptomatologies was nonopioid analgesics (NSAIDS or paracetamol) in 146 cases (66\%), weak opioids (tramadol), and strong opioids (morphine) in $8(4 \%)$ cases.

Of the 222 patients who were prescribed analgesics in the moderate pain group $(n=43 ; 19.37 \%) 19$ received NSAIDs, 5 received NSAIDs plus adjuvants, and 19 were treated with other drugs. Of the patients reporting severe pain $(n=168$; $75.68 \%$ ), 115 were treated with nonopioid analgesics (in 34 cases, nonopioid drugs plus adjuvants), 8 with opioids, and 45 with other drugs. No substantial differences in response to pharmacological therapy, evaluated as a reduction of NRS pain score, were revealed between males and females, although the highest average reduction was seen in females ( $=4.39$ points) compared to males ( $=3.97$ points). A reduction in NRS score was observed in 156 patients $(70.27 \%)$ on reevaluation of pain following initial treatment; this reduction was of at least

Table 2 Pain intensity at $t_{0}$ for the four main causes of ED admission

\begin{tabular}{|c|c|c|c|c|c|}
\hline \multirow[t]{2}{*}{ Category } & \multirow[t]{2}{*}{ NRS $t_{0}$} & \multicolumn{4}{|c|}{ Patients complaining of pain at admission $(n=732 ; 70.7 \%)$} \\
\hline & & $\begin{array}{l}\text { Traumatic } \\
(n=296 ; 40.44 \%)\end{array}$ & $\begin{array}{l}\text { Urological } \\
(n=99 ; 13.52 \%)\end{array}$ & $\begin{array}{l}\text { Abdominal } \\
(n=98 ; 13.39 \%)\end{array}$ & $\begin{array}{l}\text { Nontraumatic muscoloskeletal } \\
(\mathrm{n}=52 ; 7.10 \%)\end{array}$ \\
\hline \multirow[t]{3}{*}{ Slight pain } & I & 11 & 1 & 1 & 0 \\
\hline & 2 & 9 & 3 & 0 & I \\
\hline & 3 & 17 & 3 & 0 & 0 \\
\hline \multirow[t]{3}{*}{ Moderate pain } & 4 & 22 & 3 & 7 & 2 \\
\hline & 5 & 34 & 7 & 5 & 3 \\
\hline & 6 & 67 & 11 & 8 & 6 \\
\hline \multirow[t]{4}{*}{ Severe pain } & 7 & 69 & 22 & 21 & 11 \\
\hline & 8 & 38 & 22 & 32 & 14 \\
\hline & 9 & 16 & 12 & 16 & 7 \\
\hline & 10 & 13 & 15 & 8 & 8 \\
\hline NRS mean & & 6.08 & 7.24 & 7.44 & 7.56 \\
\hline NRS median & & 6 & 7 & 8 & 8 \\
\hline
\end{tabular}

Abbreviations: ED, emergency department; NRS, Numerical Rating Scale; $\mathrm{t}_{0}$; the first admission at triage.

Table 3 Overview of drugs employed: dose and route and frequency of administration

\begin{tabular}{lllll}
\hline Drug type & Drug used & Dose & Route of administration & Frequency of administration \\
\hline Analgesics & Paracetamol & $\mathrm{Ig}$ & Intravenous & $3 \mathrm{I}$ \\
& Diclofenac & $75 \mathrm{mg}$ & Intramuscular & 8 \\
& Ketoprofene & $100 \mathrm{mg}$ & Intravenous & 75 \\
& Ketorolac & $30 \mathrm{mg}$ & Intravenous & 38 \\
& Tramadol & $100 \mathrm{mg}$ & Intravenous & 7 \\
Adjuvants & Morphine & $10 \mathrm{mg}$ & Intramuscular & $3 \mathrm{I}$ \\
& Hydrocortisone & $100 \mathrm{mg}$ & Intravenous & 17 \\
& Betametasone & $4 \mathrm{mg}$ & Intravenous & 2 \\
& Diazepam & $2-4 \mathrm{mg}$ & Oral & 6 \\
& Clorphenamine & $10 \mathrm{mg}$ & Intramuscular & 28 \\
& Floroglucinol & $40 \mathrm{mg}$ & Intravenous & 16 \\
& Levosulpiride & $25 \mathrm{mg}$ & Intravenous & 2 \\
& Metoclopramide & $10 \mathrm{mg}$ & Intravenous & 34 \\
\hline
\end{tabular}


2 points in $84 \%$ of patients. Forty-nine individuals (22.07\%) were given second doses of analgesics. No side effects to the prescribed drugs were observed during the study in any patient.

The evaluation NRS at $\mathrm{t}_{0}$ and at discharge in the four main causes of pain is shown in Table 4.

\section{Discussion}

A prevalence of pain of $70.7 \%$ in the ED is revealed from our study, which is similar to that reported in literature, ${ }^{3,4}$ although several studies were performed over a subtype of patient and pain (eg, posttraumatic pain). The 7-day prospective study by Johnston et al ${ }^{1}$ evaluated pain prevalence and intensity in two EDs and reported a prevalence among adults equal to $71 \%$. A study by Tanabe and Buschmann ${ }^{2}$ on 203 patients aged $\geq 18$ years obtained a prevalence of $78 \%$. In another study on 726 subjects, $78 \%$ complained of pain on admission. ${ }^{7}$ These were prospective studies that not only evaluated pain prevalence but also severity and efficacy of therapy in different groups of patient complaining pain from different causes.

The importance of prospective studies derives from the inability of retrospective studies to assess pain severity, therapy response, ${ }^{2}$ or whether the pain was clinically important at the time as the opportunity to interact with the patient, the only person who can give a valid indication of his pain, no longer exists at the moment of data collection.

In an emergency setting, one-dimensional pain intensity evaluation scales are employed for their simplicity of application as they are extremely user-friendly for the majority of patients. ${ }^{15}$ The Visual Analogical Scale and the NRS of acute pain are the most widely used and validated in emergency situations and have proven to be more reliable than the Visual Rating Scale. ${ }^{16}$ The use of evaluation scales on admission to an ED and during the course of therapy appears to allow doctors to monitor the situation better and modulate analgesia, offering notable improvement in pain management in emergencies. ${ }^{17}$ Although trying to translate a number into a category like slight, moderate, or severe is probably not straightforward, this seems to be necessary in order to draft useful pain protocols. ${ }^{8-18}$

Of the 684 patients who visited the ED effectively suffering from pain, only $222(32.46 \%)$ were given pharmacological therapy. This mirrors data that has been reported in literature, or rather, that $40 \%-70 \%$ of users who access the ED due to pain do not receive analgesia or are offered inadequate treatment. ${ }^{5,19,20}$

In our study, $11.48 \%$ of patients complaining of pain left the ED before seeing a clinician or before discharge; this result is higher than the $6 \%-9 \%$ reported in literature. ${ }^{21}$ One

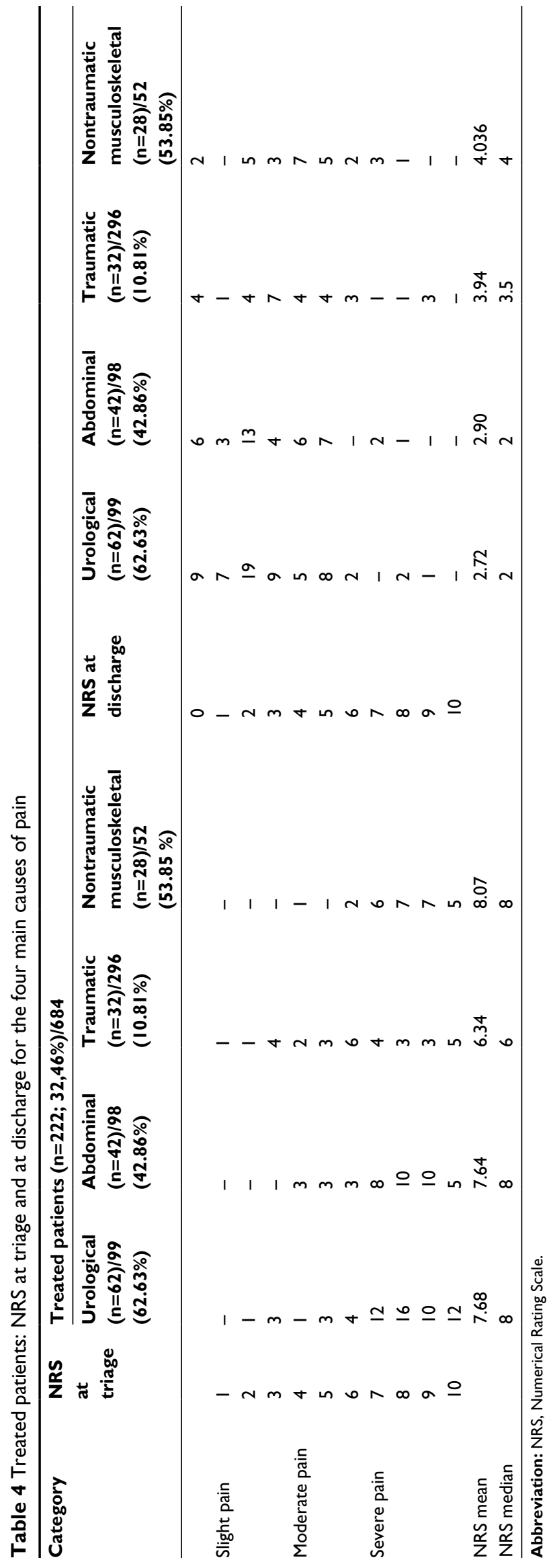


of the causes of this can be found in the frequent overcrowding of the ED, which often means a failure in the service offered because of the long waiting times and reduces drastically patient satisfaction levels. ${ }^{13,22,23,24}$

The reasons for undertreatment and inadequate management of pain therapy in ED include underevaluation and scarce accuracy during assessment; in fact, the intensity of the reported pain is often considered exaggerated by the nurses at triage. ${ }^{25,26}$

These situations could be improved with the routine registration of pain intensity by using Visual Analog Scale/ NRS at the moment of triage and with precocious analgesia protocols. ${ }^{8,19,20}$ Another cause of oligoanalgesia is the absence of specific pain management courses; ${ }^{26}$ specific training is necessary to improve the knowledge and the attitudes of health professionals toward the management and treatment of patients' pain. ${ }^{27}$ The untreated pain still remains an unmet need in ED. All analgesic drugs currently used in ED show some weak points (oral formulation with slow onset of action, need of monitoring patients receiving opioids) and also have side effects or contraindications (eg, NSAIDs in patients with cardiovascular risk).

Inhaled analgesics are not commonly used in Italy. In spite of clinical benefits, nitric oxide $\left(\mathrm{N}_{2} \mathrm{O}\right)$ use is limited by its poor practicality (considerable weight and size of the tank), risk of cross-contamination, and occupational exposure.

Methoxyflurane is an inhaled analgesic supported by recent clinical data confirming its efficacy and tolerability; ${ }^{28,29}$ it has been extensively used since the 1970s in Australia and is currently available in some European countries (UK and Ireland). Since it shows a fast onset and offset of action and is self-administered through a portable, lightweight, and easy-to-use device, it could be an advantageous option in emergency setting.

The conviction that the treatment of pain can interfere with diagnostic tests still persists today, ${ }^{30}$ or that the prescription of opioids can favor addiction. ${ }^{26}$

This was underlined by Marinangeli et al, ${ }^{31}$ in 2009, in a study on the use of analgesics in prehospital emergency situations in which they concluded that all emergency ambulances should be equipped with opioids and other analgesics for the treatment of pain. There are no "best opioids" in acute pain treatment, but some opioids are better than others in some patients. The opioids present different pharmacokinetic and pharmacodynamic properties; thus, opioid choice must be tailored for each patient.

A reduction in pain intensity of at least 2 points on the NRS scale, the value that corresponds to the cutoff score for pain necessary for an important result from a clinical viewpoint, was reported by $84 \%$ of the treated patients. ${ }^{10,16}$ Of the 296 patients who complained of pain following traumatic events, only 32 (10.81\%) were given analgesics and no opioids, even though 15 reported severe pain. This contrasts with data reported in other studies. ${ }^{17}$ This study shows that 98 patients complained of abdominal pain, and of these, only 42 were given analgesics, reporting a significant average reduction of 4.3 points in pain score, which is in accordance with the results of Farrar et al. ${ }^{10}$

Our results show that pain relief can be achieved with no opioid drugs as well.

Although a number of emergency doctors maintain that the use of painkillers does not compromise medical examination, most procrastinate analgesia until the patient's diagnostic evaluation is completed. . $^{32,33}$

Diagnosis and treatment of pain should be performed as speedily as possible and begin, where possible and safe, before arrival in hospital. ${ }^{31}$ Analgesia in the ED is still largely inadequate today $;^{7,11,34}$ in order to obviate this, we believe it is fundamental that emergency doctors fully understand the pathophysiological implications of acute pain, which not only signifies sufferance for the patient but is also the cause of functional alteration of numerous apparatus including the cardiovascular and endocrinal systems. Inadequate treatment of acute pain can aggravate the clinical pictures of the more critical patients and those less able to adapt to a worsening of their illness (the elderly, cardiopaths, cancer patients, diabetics, etc.). In consideration of this, it could be useful to intensify pain treatment training and to create flexible protocols for the treatment of acute pain in ED.

Correct pain management requires knowledge about pharmacological properties of opioids, in particular the assessment of risks related to opioid diversion, abuse, and misuse. ${ }^{35-36}$

In this setting, pain treatment protocols should be implemented at the time of triage, immediately after patient's admission in the ED. Patients should be frequently asked about their pain score through validated pain score scales, as we usually do with other vital signs, and the score should be registered step by step into the patient's clinical record. ${ }^{5,12,20}$

\section{Limitations}

A number of factors limit our study. First of all, this is a single-center study, and a 4-week duration is a short time in order to obtain a global view about ED pain. Another limitation is the lack of data about a possible prehospital pain treatment. 
Unfortunately, nonpharmacological pain control methods, including psychosocial treatment, were not evaluated in this work.

\section{Conclusion}

This study confirms that pain, whatever its etiology, represents one of the principal reasons for visit to and treatment at an ED. Despite significant reduction in pain levels in recipients of analgesics, our study shows that undertreatment of pain in the ED is still a problem in need of solutions.

\section{Disclosure}

The authors report no conflicts of interest in this work.

\section{References}

1. Johnston CC, Gagnon AJ, Fullerton L, et al. One-week survey of pain intensity on admission to and discharge from the emergency department: a pilot study. J Emerg Med. 1998;16(3):377-382.

2. Tanabe $P$, Buschmann M. A prospective study of ED pain management practices and the patient's perspective. J Emerg Nurs. 1999;25(3): 171-177.

3. Allen MA, Jewers H, McDonald JS, et al. A framework for the treatment of pain and addiction in the emergency department. J Emerg Nurs. 2014;40(6):552-559

4. Chang HY, Daubresse M, Kruszewski SP, Alexander GC. Prevalence and treatment of pain in EDs in the United States, 2000 to 2010. Am J Em Med. 2014;(32):421-431.

5. Lynch ME. The need for a Canadian pain strategy. Pain Res Manag. 2011;16(2):77-80.

6. Dale J, Bjornsen LP. Assessment of pain in a Norwegian Emergency Department. Scand J Trauma Resusc Emerg Med. 2015;23:86.

7. Tcherny-Lessenot S, Karwowski-Soulié F, Lamarche-Vadel A, et al. Management and relief of pain in an emergency department from the adult patients' perspective. J Pain Symptom Manage. 2003;25(6):539-546.

8. Savoia G, Coluzzi F, Di Maria C, et al. Italian Intersociety Recommendations on pain management in the emergency setting (SIAARTI, SIMEU, SIS 118, AISD, SIARED, SICUT, IRC). Minerva Anestesiol. 2015;81(2):205-225.

9. Wilson JE, Pendleton JM. Oligoanalgesia in the emergency department. Am J Emerg Med. 1989;7(6):620-623.

10. Farrar JT, Berlin JA, Strom BL. Clinically important changes in acute pain outcome measures: a validation study. J Pain Symptom Manage. 2003;25(5):406-411.

11. Fosnocht DE, Swanson ER, Barton ED. Changing attitudes about pain and pain control in emergency medicine. Emerg Med Clin North Am. 2005;23(2):297-306.

12. Stang AS, Hartling L, Fera C, Johnson D, Ali S. Quality indicators for the assessment and management of pain in the emergency department: a systematic review. Pain Res Manag. 2014;19(6): e179-e190.

13. Fosnocht DE, Heaps ND, Swanson ER. Patient expectations for pain relief in the ED. Am J Emerg Med. 2004;22(4):286-288.

14. Hatherley C, Jennings N, Cross R. Time to analgesia and pain score documentation best practice standards for the Emergency Department-A literature review. Australas Emerg Nurs J. 2016;19(1):26-36.
15. Breivik H, Borchgrevink PC, Allen AM, et al. Assessment of pain. $\mathrm{Br}$ J Anaesth. 2008;101(1):17-24.

16. Bijur PE, Latimer CT, Gallagher EJ. Validation of a verbally administered numerical rating scale of acute pain for use in the Emergency Department. Acad Emerg Med. 2003;10(4):390-392.

17. Silka PA, Mendel MR, Moreno G, et al. Pain scores improve analgesic administration patterns for trauma patients in the emergency department. Acad Emerg Med. 2004;11(3):264-270.

18. Boonstra AM, Stewart RE, Köke AJ, et al. Cut-off points for mild, moderate and severe pain on numeric rating scale for pain in patients with chronic musculoskeletal pain: variability and influence of sex and catastrophizing. Front Psychol. 2016;7:1466.

19. Stalnikowicz R, Mahamid R, Kaspi S, et al. Undertreatment of acute pain in the emergency department: a challenge. Int J Qual Health Care. 2005;17(2):173-176.

20. Guéant S, Taleb A, Borel-Kühner J, et al. Quality of pain management in the emergency department: results of a multicentre prospective study. Eur J Anaesthesiol. 2011;28(2):97-105.

21. Wheeler E, Klemm P, Shonewolf E. Level of pain and waiting time in the emergency department. Pain Manag Nurs. 2010;11(2):108-114.

22. Arendts G, Fry M. Factors associated with delay to opiate analgesia in emergency departments. J pain. 2006;7(9):682-686.

23. Rhodes $\mathrm{K}$. The effect of crowding on access and quality in an academic ED. Am J Emerg Med. 2006;24(7):787-794.

24. Todd KH, Ducharme J, Choiniere M. Pain in the emergency department: results of the pain and emergency medicine (PEMI) multicenter study. J Pain. 2007;8(6):460-466.

25. Guru V, Dubinsky I. The patient versus caregiver perception of acute pain in the emergency department. J Emerg Med. 2000;18(1):7-12.

26. Rupp T, Delaney KA. Inadequate analgesia in emergency medicine. Ann Emerg Med. 2004;43(4):494-503.

27. Visentin M, Zanolin E, Trentin L, et al. Prevalence and treatment of pain in adults admitted to Italian hospitals. Eur J Pain. 2005;9(1):61-67.

28. Blair HA, Frampton JE. Methoxyflurane: a review in trauma pain. Clin Drug Investig. 2016;36(12):1067-1073.

29. Coffey F, Wright J, Hartshorn S, et al. STOP!: a randomised, doubleblind, placebo-controlled study of the efficacy and safety of methoxyflurane for the treatment of acute pain. Emerg Med J. 2014;31(8): 613-618.

30. Thomas SH, Silen W, Cheema F, et al. Effects of morphine analgesia on diagnostic accuracy in emergency department patients with abdominal pain: a prospective, randomized trial. J Am Coll Surg. 2003;196(1): v18-31.

31. Marinangeli F, Narducci C, Ursini ML, et al. Acute pain and availability of analgesia in the pre-hospital emergency setting in Italy: a problem to be solved. Pain Prac. 2009;9(4):282-288.

32. Wolfe JM, Lein DY, Lenkoski K, et al. Analgesic administration to patients with an acute abdomen: a survey of emergency medicine physicians. Am J Emerg Med. 2000;18(3):250-253.

33. Yee AM, Puntillo K, Miaskowski C, et al. What patients with abdominal pain expect about pain relief in the emergency department. $J$ Emerg Nurs. 2006;32(4):281-287.

34. Grant PS. Analgesia delivery in the ED. Am J Emerg Med. 2006;24(7); 806-809.

35. Cantrill SV, Brown MD, Carlisle RJ, et al. Clinical policy: critical issues in the prescribing of opioids for adult patients in the emergency department. Ann Emerg Med. 2012;60(4):499-525.

36. Young HW, Tyndall JA, Cottler LB. The current utilization and perceptions of prescription drug monitoring programs among emergency medicine providers in Florida. Int J Emerg Med. 2017;10:16. 
The Journal of Pain Research is an international, peer reviewed, open access, online journal that welcomes laboratory and clinical findings in the fields of pain research and the prevention and management of pain. Original research, reviews, symposium reports, hypothesis formation and commentaries are all considered for publication.
The manuscript management system is completely online and includes a very quick and fair peer-review system, which is all easy to use. Visit http://www.dovepress.com/testimonials.php to read real quotes from published authors. 